\title{
THE CALCULATION OF LEAKED ELECTROMAGNETIC FIELD IN GALLERY OF KLYSTRON OF NSRL
}

\author{
Tao Xiaoping, Wang Guicheng, NSRL, USTC, China
}

\section{Abstract}

The HF microwave leak in the gallery of klystron of NSRL affects microwave communication greatly. In order to reduce electromagnetic radiation noise, it is necessary to locate the noise source. But for the complicated leaked field, it is difficult to locate the noise source. We can test field and calculate it, so as to locate the radiation noise source and study the method of reduction of the electromagnetic noise. This article gives the method and formulae of calculation of electromagnetic field, and also contrast test.

\section{THE RADIATION FIELD OF APERTURE ANTENNA $[1,2]$}

There are several apertures on the modulator shielding tank, which radiate HF electromagnetic field. The radiation field is calculated using the model of aperture antenna.

Providing that the noise source $\left(E_{n}=E_{n m} \sin \left[k_{n}\left(L_{n}-\left|z_{n}^{\prime}\right|\right)\right] e^{-i\left(\omega_{n} t+\varphi_{n}\right)}\right)$ is situated on $\left(x_{n 0}, y_{n 0}, z_{n 0}\right)$, the direction cosine is $\left(\cos \alpha_{n o}, \cos \beta_{n 0}, \cos \gamma_{n 0}\right)$, the source frequency is $\omega_{n}\left(k_{n}=\frac{\omega_{n}}{c}\right)$, and $L_{n}$ is the half-length of aperture antenna, $E_{n m}$ is electric field amplitude in center of the aperture, $d_{n}$ is width of the aperture. According to the principle of duality: $\vec{E} \leftrightarrow \vec{H}, \varepsilon_{0} \leftrightarrow \mu_{0}$, the radiation field is deduced.

That is

$$
\begin{aligned}
\vec{E}_{n}= & -K_{n} \sin \left(k_{n} R_{n}-\omega_{n} t-\varphi_{n}\right) \\
{[} & \left(\cos \beta_{n} \cos \gamma_{n 0}-\cos \gamma_{n} \cos \beta_{n 0}\right) \vec{e}_{x} \\
& +\left(\cos \gamma_{n} \cos \alpha_{n 0}-\cos \alpha_{n} \cos \gamma_{n 0}\right) \vec{e}_{y} \\
& \left.+\left(\cos \alpha_{n} \cos \beta_{n 0}-\cos \beta_{n} \cos \alpha_{n 0}\right) \vec{e}_{z}\right]
\end{aligned}
$$

The magnitude of $\vec{E}$ is

$$
\begin{aligned}
& E(t)=\left\{\left[-\sum_{1}^{n} K_{n} \cdot \sin \left(k_{n} R_{n}-\omega_{n} t-\varphi_{n}\right) .\right.\right. \\
& \left.\quad\left(\cos \beta_{n} \cos \gamma_{n 0}-\cos \gamma_{n} \cos \beta_{n 0}\right)\right]^{2} \\
& +\left[-\sum_{1}^{n} K_{n} \cdot \sin \left(k_{n} R_{n}-\omega_{n} t-\varphi_{n}\right) .\right. \\
& \left.\left(\cos \gamma_{n} \cos \alpha_{n 0}-\cos \alpha_{n} \cos \gamma_{n 0}\right)\right]^{2} \\
& \quad+\left[-\sum_{1}^{n} K_{n} \cdot \sin \left(k_{n} R_{n}-\omega_{n} t-\varphi_{n}\right) .\right.
\end{aligned}
$$

$$
\left.\left.\left(\cos \alpha_{n} \cos \beta_{n 0}-\cos \beta_{n} \cos \alpha_{n 0}\right)\right]^{2}\right\}^{1 / 2}
$$

If period of the $E$ is $T$, basing the equation $E(t)=E(t+T)$, the $T$ can be got.

The effective value of $E(t)$ is

$$
E_{\text {effect }}=\sqrt{\frac{1}{T} \int_{0}^{T} E^{2}(t) d t} .
$$

Where

$$
\begin{gathered}
K_{n}=\frac{E_{n m} d_{n}}{\pi R_{n}} \cdot \frac{\cos \left(\frac{\omega_{n} L_{n}}{c} \cos \theta_{n}\right)-\cos \left(\frac{\omega_{n} L_{n}}{c}\right)}{\sin ^{2} \theta_{n}}, \\
R_{n}=\sqrt{\left(x-x_{n 0}\right)^{2}+\left(y-y_{n 0}\right)^{2}+\left(z-z_{n 0}\right)^{2}}, \\
\cos \alpha_{n}=\frac{x-x_{n 0}}{R_{n}}, \cos \beta_{n}=\frac{y-y_{n 0}}{R_{n}}, \cos \gamma_{n}=\frac{z-z_{n 0}}{R_{n}},
\end{gathered}
$$

$\cos \theta_{n}=\cos \alpha_{n} \cos \alpha_{n 0}+\cos \beta_{n} \cos \beta_{n 0}+\cos \gamma_{n} \cos \gamma_{n 0}$, $\sin \theta_{n}=\sqrt{1-\left(\cos \alpha_{n} \cos \alpha_{n 0}+\cos \beta_{n} \cos \beta_{n 0}+\cos \gamma_{n} \cos \gamma_{n 0}\right)^{2}}$.

\section{THE RADIATION FIELD OF \\ TRAVELLING WAVE ANTENNA}

The electromagnetic radiation of transmission cable of modulator is calculated using the model of travelling wave antenna. Supposing there is electric current $I_{z}=I_{0} e^{i(k z-\omega t)}$ in line antenna, and the antenna length is $2 L$ (see Fig1), the radiation vector potential $\vec{A}$ is[3]

$$
A_{z}=-\mu \frac{e^{i k R}}{4 \pi R} \cdot \frac{2 I_{0} \sin [k L(1-\cos \theta)]}{k(1-\cos \theta)}
$$

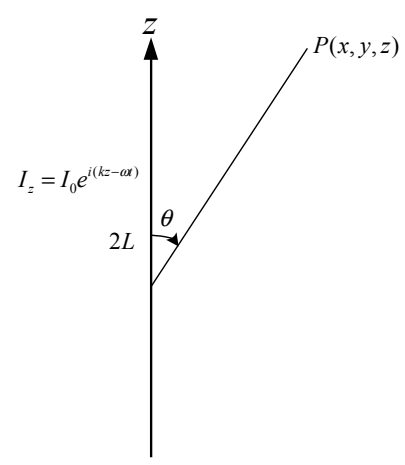

Figure 1: The model of travelling wave antenna.

If the antenna is situated on $\left(x_{0}, y_{0}, z_{0}\right)$, and the direction cosine is $\left(\cos \alpha_{0}, \cos \beta_{0}, \cos \gamma_{0}\right)$ the radiation vector potential $\vec{A}$ is 
$\vec{A}=-\mu I_{0} \frac{e^{i k R}}{2 \pi R} \cdot \frac{\sin [k L(1-\cos \theta)]}{k(1-\cos \theta)}\left(\cos \alpha_{0} \vec{e}_{x}+\cos \beta_{0} \vec{e}_{y}+\cos \gamma_{0} \vec{e}_{z}\right)$

For $\vec{B}=\nabla \times \vec{A}, \vec{E}=-c \vec{n} \times \vec{B}$, So

$\vec{B}=\nabla \times \vec{A}=i k \vec{n} \times \vec{A}=i k\left(\cos \alpha \vec{e}_{x}+\cos \beta \vec{e}_{y}+\cos \vec{e}_{z}\right) \times \vec{A}$ $=-\frac{i \mu I_{0} e^{i k R}}{2 \pi R} \cdot \frac{\sin [k L(1-\cos \theta)]}{1-\cos \theta}\left[\left(\cos \beta \cos \gamma_{0}-\cos \gamma \cos \beta_{0}\right) \vec{e}_{x}\right.$

$\left.+\left(\cos \gamma \cos \alpha_{0}-\cos \alpha \cos \gamma_{0}\right) \vec{e}_{y}+\left(\cos \alpha \cos \beta_{0}-\cos \beta \cos \alpha_{0}\right) \vec{e}_{z}\right]$

$\vec{E}=-c \vec{n} \times \vec{B}=\frac{i \mu I_{0} c e^{i k R}}{2 \pi R} \cdot \frac{\sin [k L(1-\cos \theta)]}{1-\cos \theta}$

$\cdot\left[\left(\cos \beta \cos \alpha \cos \beta_{0}-\cos ^{2} \beta \cos \alpha_{0}-\cos ^{2} \gamma \cos \alpha_{0}+\cos \gamma \cos \alpha \cos \gamma_{0}\right) \vec{e}_{x}\right.$

$+\left(\cos \gamma \cos \beta \cos \gamma_{0}-\cos ^{2} \gamma \cos \beta_{0}-\cos ^{2} \alpha \cos \beta_{0}+\cos \alpha \cos \beta \cos \alpha_{0}\right) \vec{e}_{y}$ $\left.+\left(\cos \alpha \cos \gamma \cos \alpha_{0}-\cos ^{2} \alpha \cos \gamma_{0}-\cos ^{2} \beta \cos \gamma_{0}+\cos \beta \cos \gamma \cos \beta_{0}\right) \vec{e}_{z}\right]$

The real part is

$\vec{B}=\frac{\mu I_{0} \sin k R}{2 \pi R} \cdot \frac{\sin [k L(1-\cos \theta)]}{1-\cos \theta}\left[\left(\cos \beta \cos \gamma_{0}-\cos \gamma \cos \beta_{0}\right) \vec{e}_{x}\right.$

$\left.+\left(\cos \gamma \cos \alpha_{0}-\cos \alpha \cos \gamma_{0}\right) \vec{e}_{y}+\left(\cos \alpha \cos \beta_{0}-\cos \beta \cos \alpha_{0}\right) \vec{e}_{z}\right]$

$\vec{E}=-\frac{\mu I_{0} c \sin k R}{2 \pi R} \cdot \frac{\sin [k L(1-\cos \theta)]}{1-\cos \theta}$

. $\left[\left(\cos \beta \cos \alpha \cos \beta_{0}-\cos ^{2} \beta \cos \alpha_{0}-\cos ^{2} \gamma \cos \alpha_{0}+\cos \gamma \cos \alpha \cos \gamma_{0}\right) \vec{e}_{x}\right.$

$+\left(\cos \gamma \cos \beta \cos \gamma_{0}-\cos ^{2} \gamma \cos \beta_{0}-\cos ^{2} \alpha \cos \beta_{0}+\cos \alpha \cos \beta \cos \alpha_{0}\right) \vec{e}_{y}$

$\left.+\left(\cos \alpha \cos \gamma \cos \alpha_{0}-\cos ^{2} \alpha \cos \gamma_{0}-\cos ^{2} \beta \cos \gamma_{0}+\cos \beta \cos \gamma \cos \beta_{0}\right) \vec{e}_{z}\right]$

Where

$$
\begin{aligned}
& R=\sqrt{\left(x-x_{0}\right)^{2}+\left(y-y_{0}\right)^{2}+\left(z-z_{0}\right)^{2}} \\
& \cos \alpha=\frac{x-x_{0}}{R}, \cos \beta=\frac{y-y_{0}}{R}, \cos \gamma=\frac{z-z_{0}}{R}, \\
& \cos \theta=\cos \alpha \cos \alpha_{0}+\cos \beta \cos \beta_{0}+\cos \gamma \cos \gamma_{0}, \\
& \sin \theta=\sqrt{1-\left(\cos \alpha \cos \alpha_{0}+\cos \beta \cos \beta_{0}+\cos \gamma \cos \gamma_{0}\right)^{2}} .
\end{aligned}
$$

\section{THE RADIATION FIELD OF ROTUNDITY PLANE CALIBER ANTENNA}

There are eight roundity ventholes on the top of modulator, which radiate outwards electromagnetic noise during pulse discharging. The radiation field of the ventholes is calculated using the model of rotundity plane caliber antenna[4].

\subsection{The radiation of basic surface-element}

Supposing the basic small surface-element is situated on origin of coordinates, the radiation electric field intensity $d E_{\theta}$ and $d E_{\varphi}$ of Huigensi element are

$$
\begin{array}{r}
d E_{\theta}=-j \frac{E_{0 x} d s}{2 \lambda r}(1+\cos \theta) \cos \varphi e^{-j k r}, \\
d E_{\varphi}=-j \frac{E_{0 x} d s}{2 \lambda r}(1+\cos \theta) \sin \varphi e^{-j k r} \\
d \vec{E}=d E_{\theta} \vec{e}_{\theta}+d E_{\varphi} \vec{e}_{\varphi}=-j \frac{E_{0 x} d s}{2 \lambda r}(1+\cos \theta) . \\
\cos \varphi e^{-j k r} \vec{e}_{\theta}-j \frac{E_{0 x} d s}{2 \lambda r}(1+\cos \theta) \sin \varphi e^{-j k r} \vec{e}_{\varphi}
\end{array}
$$

where

$$
\begin{aligned}
& r=\sqrt{x^{2}+y^{2}+z^{2}}, \cos \theta=\frac{z}{r}, \sin \theta=\frac{\sqrt{r^{2}-z^{2}}}{r}, \\
& \cos \varphi=\frac{x}{\sqrt{r^{2}-z^{2}}}, \sin \varphi=\frac{y}{\sqrt{r^{2}-z^{2}}}
\end{aligned}
$$

So the radiation electric field is

$d \vec{E}=-\frac{E_{0 x} d s}{2 \lambda r} \sin k r$.

$$
\begin{aligned}
& \quad\left\{\left[\frac{x^{2} z}{r\left(r^{2}-z^{2}\right)}+\frac{x^{2} z^{2}}{r^{2}\left(r^{2}-z^{2}\right)}-\frac{y^{2}}{r^{2}-z^{2}}-\frac{y^{2} z}{r\left(r^{2}-z^{2}\right)}\right] \vec{e}_{x}\right. \\
& +\left[\frac{2 x y z}{r\left(r^{2}-z^{2}\right)}+\frac{x y z^{2}}{r^{2}\left(r^{2}-z^{2}\right)}+\frac{x y}{r^{2}-z^{2}}\right] \vec{e}_{y} \\
& \left.-\left[\frac{x}{r}+\frac{x z}{r^{2}}\right] \vec{e}_{z}\right\}
\end{aligned}
$$

\subsection{The radiation of rotundity plane caliber antenna}

Because the plane caliber antenna is very small, the amplitude of electric field and the phase of the antenna are homogeneous distribution. So

$\vec{E}=\int d \vec{E}=-\frac{\pi E_{0 x} D^{2}}{2 \lambda r^{2}\left(r^{2}-z^{2}\right)} \sin k r$

$\left\{\left(x^{2} z+\frac{x^{2} z^{2}}{r}-r y^{2}-y^{2} z\right) \vec{e}_{x}+\left(2 x y z+\frac{x y z^{2}}{r}+r x y\right) \vec{e}_{y}\right.$

$\left.-\left(x+\frac{x z}{r}\right)\left(r^{2}-z^{2}\right) \vec{e}_{z}\right\}$

\subsection{The general formulae of rotundity plane caliber antenna}

If the centre position is situated on $\left(x_{0}, y_{0}, z_{0}\right)$, the general calculation formula of radiation field is

$$
\begin{aligned}
\vec{E}=-\frac{\pi E_{0 x} D^{2}}{2 \lambda r^{2}\left[r^{2}-\left(z-z_{0}\right)^{2}\right]} \sin \frac{2 \pi}{\lambda} r . \\
\left\{\left[\left(x-x_{0}\right)^{2}\left(z-z_{0}\right)+\frac{\left(x-x_{0}\right)^{2}\left(z-z_{0}\right)^{2}}{r}-r\left(y-y_{0}\right)^{2}-\left(y-y_{0}\right)^{2}\left(z-z_{0}\right)\right] \vec{e}_{x}\right. \\
+\left[2\left(x-x_{0}\right)\left(y-y_{0}\right)\left(z-z_{0}\right)+\frac{\left(x-x_{0}\right)\left(y-y_{0}\right)\left(z-z_{0}\right)^{2}}{r}+r\left(x-x_{0}\right)\left(y-y_{0}\right)\right] \vec{e}_{y}
\end{aligned}
$$


$\left.-\left[\left[x-x_{0}+\frac{\left(x-x_{0}\right)\left(z-z_{0}\right)}{r}\right] \cdot\left[r^{2}-\left(z-z_{0}\right)^{2}\right]\right] \vec{e}_{z}\right\}$

Where

$$
r=\sqrt{\left(x-x_{0}\right)^{2}+\left(y-y_{0}\right)^{2}+\left(z-z_{0}\right)^{2}},
$$

$E_{0 x}$ is the amplitude of electric field of the basic surface-element,

$D$ is the semi-diameter and $\lambda$ is wave-length of radiation electromagnetic wave.

\section{Simulation CALCULATION AND CONTRAST TEST[5]}

\subsection{The noise distribution of one modulator along gallery direction ( $y$ direction)}

In order study on the distribution of electromagnetic noise in klystron gallery, the noise voltage along klystron gallery direction is calculated and tested. When high voltage of the second modulator is $10 \mathrm{KV}$, the test and calculation result is showed as curve A and B (see Fig.2).

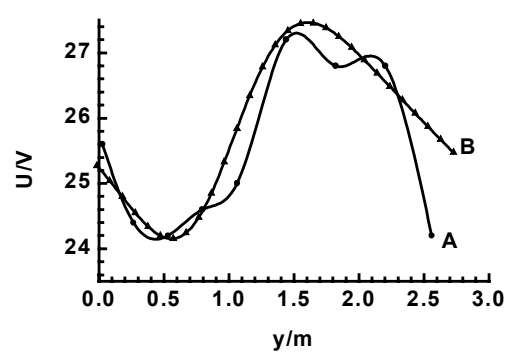

Figure 2: Simulation computation and test curves of leaked noise along $y$ direction.

\subsection{The noise distribution of one modulator along being off modulator direction ( $x$ direction)}

Fig. 3 shows the noise voltage distribution of one modulator along being off modulator direction, which curve $\mathrm{A}$ is the tested noise voltage and curve $\mathrm{B}$ is the calculation result.

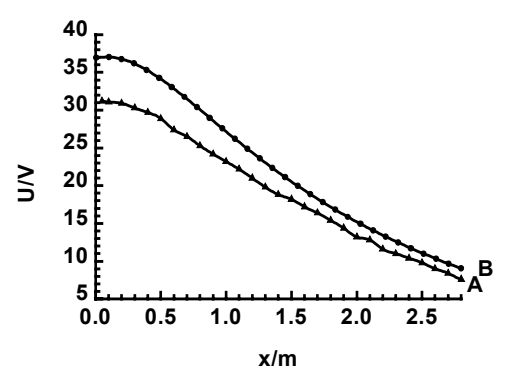

Figure 3: Simulation computation and test curves of leaked noise along $x$ direction.

\subsection{The noise distribution of five modulator along gallery direction ( $y$ direction)}

Under the situation of half-voltage and half-power of all five modulator, the contrast tests are made. The curve A and B in Fig.4 are test and calculation curves when these modulator are in high-voltage. The small rectangles in Fig. 4 are in turn reference situations of No.1 to No.5 modulator.

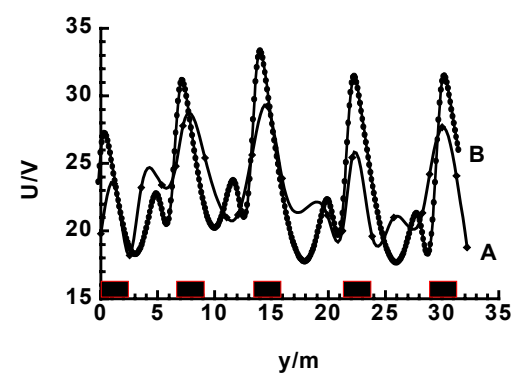

Figure 4: Simulation computation and test curves of leaked noise.

\section{CONCLUSION}

This article gives theoretical calculation about radiation electromagnetic noise in the klystron gallery.

According to the discharging characteristics of high voltage pulse modulator and the structure of modulator, we have built physics models about radiation noise source in the klystron gallery - aperture antenna rotundity plane caliber antenna and travelling wave antenna. In the right-angle coordinate system, we have deduced the calculation formulae about radiation electromagnetic field in the klystron gallery. Using them, we have compiled calculation process about radiation electromagnetic field and calculated the distribution of radiation noise in the klystron gallery. The result of on-the-spot contrast testing shows rationality of the simulation calculation which offers theoretical foundation for the reduction of electromagnetic radiation noise.

\section{REFERENCES}

[1] WAN Wei, WANG Jili. Microwave Technology and Antenna (in Chinese). Xi'an: Publishing House of Industry University of West and North, 1986,184-186,276-279,208-210

[2] LOU Renhai, FU Guoxin, XIAO Shujun. Electromagnetic Theory of Engineer(in Chinese). Beijing: Publishing House of National Defense Industry, 1991,149-155

[3] JIN Yaqiu. Electromagnetic Waves in Complicated System(in Chinese). Shanghai: Publishing House of Fudan, 1995, 236-247

[4] MITTRA R. Computer Techniques for Electromagnetics.1973,89-96

[5] Ott, Henry W. Noise Reduction Techniques in electronic systems.1976,128-141 